\title{
Reducing time to reperfusion for ST elevation myocardial infarction patients by a simple process change in the Emergency Department
}

Jill Byrne

Belfast Health and Social care Trust

\begin{abstract}
Current reperfusion guidelines from the European Society of Cardiology (ESC) recommend that First Medical Contact to Balloon times (FMCB) for ST elevation myocardial infarction (STEMI) should not exceed 120 minutes. Many factors can cause delay in door to balloon times for patients suffering from STEMI. Previous studies have found that longest times for FMC-B occur if the patient presents initially to a nonpercutaneous intervention $(\mathrm{PCl})$ capable hospital. As a non- $\mathrm{PCl}$ capable site we looked at ways of reducing FMC-B times. Audit revealed that registration to electrocardiogram (ECG) times were sometimes prolonged due to undertriage, long waiting times and lack of space and staff to record an ECG, resulting in some prolonged FMC-B times.
\end{abstract}

To address this, we have changed the system so that patients bypass triage and go directly to a dedicated investigation cubicle for an ECG. The patient books on at reception with chest pain and is immediately directed to the investigation cubicle. The ECG is reviewed immediately and the patient is either kept in the department for further management or allowed back to the waiting room to await triage if the ECG is nondiagnostic and history not worrying.

Data on patients presenting with STEMI on the initial ECG were collected for one year pre-intervention $(\mathrm{n}=21)$ and one year post-intervention $(n=17)$. The median FMC-B time for the pre-intervention group was 108.5 minutes (IQR $96-143.5)$. Median FMC-B time for the postintervention group was 82 minutes (IQR 66.5 - 93.5).

This is a simple, low-cost intervention which could be transferable to other sites who have an interest in reducing FMC-B times. It is necessary to have a key person to carry out audit of all potential areas of delay, and a collaborative, multidisciplinary approach to making change to improve quality of care.

\section{Problem}

Time to reperfusion for patients suffering from ST elevation myocardial infarction (STEMI) should be as short as possible to preserve myocardium and ensure better outcomes in terms of morbidity and mortality for these patients. This is achieved by the process of primary percutaneous intervention (PPCl), where the coronary artery is opened by means of inflation of a balloon in the coronary artery affected by thrombus and deployment of a coronary stent. Time to reperfusion is referred to as first medical contact to balloon (FMC-B).

Current guidelines recommend FMC-B time should be no longer than 120 minutes (1). Therefore it is important to identify and manage these patients rapidly, however, this presents a challenge to Emergency Departments (ED) which are not attached to interventional centres. This means that transfer time has to be added to the time from first medical contact, defined as when the patient registers at reception, or the arrival of emergency services who transport the patient to the ED.

\section{Background}

The National Infarction Angioplasty Project (2) found that PPCI was superior to thrombolysis, the pharmacological method of reperfusion used previously, in terms of mortality in hospital, at 30 days and at one year. It found that PPCI was an achievable option in the United Kingdom provided acceptable times could be achieved. The fastest route to treatment for the patient is direct access to the cardiac catheterisation laboratory via the emergency services with times increasing if the patient first presents to an Emergency Department (ED) either in a PPCl-capable or non-PPCl capable hospital. Median FMC-B times were found to be highest, at 159 minutes, if the patient had to undergo inter-hospital transfer from a non-interventional centre to an interventional centre.

There are many areas where delay can occur for these patients, due to simple factors like incorrect triage, lack of space to perform an ECG or failure of the clinician to diagnose STEMI on the ECG. The most commonly used triage system in the United Kingdom is the Manchester Triage system (3). Triage is a process whereby the triage nurse takes a brief history from the patient, records clinical observations and assigns a patient to a category, according to the presenting complaint and clinical signs. This determines the priority for medical attention and further diagnostic testing (3). Patients who present with chest pain can be assigned to either an orange or yellow triage category depending on the decision the triage nurse makes on assessment. The Manchester Triage system suggests that patients triaged orange category should be seen within 10 
minutes, and those triaged as yellow should be seen within an hour. Therefore, if a patient who is presenting with a STEMI is triaged as yellow due to atypical symptoms or does not look very unwell, there may be a substantial delay in obtaining an ECG. Cooke and Jinks

(4) found that the Manchester Triage system failed to detect a small number of critically ill patients, including one patient with an MI who presented atypically. Van der Wulp et al (5) found that under-triage occurs mainly in the orange and yellow categories, which would be consistent with findings on local audit.

College of Emergency Medicine guidelines (6) and European Society for Cardiology guidelines set a standard of ECG within 10 minutes of registration for patients presenting with suspected MI[1] Delay to triage due to high volume of patients presenting to reception or by ambulance will have a knock-on effect on time taken to recording an ECG. Having a yellow triage category (can be seen within one hour) will also cause a delay to ECG. If these standards are to be met and maintained continual audit of practice must occur. One of the factors which may help improve these times is prospective audit and action as soon as it becomes apparent there has been a delay or there is a potential for delay.

\section{Baseline measurement}

Audit of ECG times was carried out on a monthly basis and results fed back to ED medical and nursing staff. Initially audit was carried out on all patients who had a triage category of "orange cardiac chest pain" as these patients were supposed to be seen as a priority within 10 minutes of triage. These initial audits allowed a baseline to be established. Initially, only $30 \%$ of patients presenting with chest pain met the target of an ECG within 10 minutes. Audit of FMC-B times was also ongoing. As delays occurred and were investigated it became apparent that a significant number of STEMI patients had been triaged under a yellow category meaning that ECG could be delayed by an hour or more.

See supplementary file: ds3328.pptx - "STEMI process map"

\section{Design}

It was then deemed necessary to include in audit all patients presenting with chest pain regardless of triage category, as potentially anyone with chest pain could turn out to be having a STEMI, but this is impossible to diagnose without an ECG. Ongoing monthly audit of patients presenting consecutively over a 72 hour period was carried out and each time results were reported back to ED nursing and medical staff. All patients with non-traumatic chest pain were included to assess how quickly an ECG was obtained as it was technically possible that any of these patients was potentially having a STEMI. No improvement was apparent under the system in place despite the best efforts of all involved.

A proposal was put forward to have an ECG recorded on all patients with chest pain as soon as they were booked on at reception and prior to triage. This was rejected at this point by the ED staff as it represented a significant change in practice and raised the question of where these patients could go for an ECG as there was no dedicated area without potentially losing an examination cubicle to achieve improved ECG times.

A further incident occurred when a STEMI patient had a long delay to ECG as the patient was allocated a yellow triage category. This resulted in delay and a subsequent long FMC-B time with left ventricular impairment which may not have occurred had the patient been diagnosed and transferred in a timely manner. A Clinical Incident form was completed and the subject tabled for further discussion at the next ED management meeting. Following prolonged discussion and debate it was agreed to trial a dedicated cubicle with a health care assistant to record ECGs before triage for a 3 day period, to audit this and then look at the data. The audit data showed some improvement but not so much as had been hoped. Times improved but there was some confusion as to whether the patient should be triaged before or after the ECG among staff, with roughly half having an ECG before triage and half after. The triage nurse has a responsibility to assess the patient personally and assign a triage category, and there was a fear that patients may be missed. Due to staffing levels, the previous system remained in place. All staff saw a need to improve times but found it hard to put the systems in place to support change.

Over the January and February of 2011, 14 patients with STEMI presented to the ED in the non-interventional site. Of these, 6 were triaged as yellow and had prolonged times to ECG. This data was shared with ED consultants and nursing staff and the decision was made to make a change in practice and to sustain it if possible. A dedicated cubicle was designated for recording ECGs. As soon as the patient booked on at reception with chest pain, they were directed to this cubicle where an ECG would be recorded and reviewed immediately. If there were concerns about the ECG the patient would be moved to a different cubicle to free up the ECG cubicle and the nurse responsible for that area would ensure the patient was triaged. If the ECG was not abnormal the patient would go back to the waiting area to be called for triage. Initially there was no designated member of staff to perform an ECG, however there is now a Health care assistant allocated to this cubicle.

\section{Strategy}

PDSA 1 showed that even though awareness had been raised and everyone was aware and trying to address the issue, space and triage issues remained.

PDSA 2 tackled the issue of space by a trial of a dedicated cubicle for ECG recording but did not deal with delays at triage or undertriage of patients with STEMI.

PDSA 3 aimed to change the process from register- triage-ECG to register-ECG-triage following review of the ECG.

See supplementary file: ds3330.docx - "STEMI pdsa 2"

\section{Post-measurement}

ECG times continued to be audited on a monthly basis looking at 


\section{BMJ Quality Improvement Reports}

the percentage of ECG times less than 10 minutes from triage. Gradually as the system became embedded these percentages began to improve. At the same time, it was noted that patients with STEMI who presented with STEMI on the initial ECG appeared to be achieving shorter FMC-B times. Analysis was carried out on all the patients who presented with STEMI on the initial ECG from March 2010- February 2011 and compared with the same group presenting from March 2011 -February 2012 giving data for one year pre-intervention and one year post-intervention. Median FMC$B$ for the pre-intervention year was 108.5 minutes for patients presenting with STEMI on the initial ECG. Median FMC-B time in the post-intervention group with STEMI present on the initial ECG was 82 minutes, a reduction of 26.5 minutes. The fact that this part of the patient journey has been reduced means that if other areas of delay do occur, there is still a strong likelihood that target times will be met.

\section{Lessons and limitations}

Carrying out baseline audit and repeating the audit cycle regularly to assess effectiveness of any interventions has produced not only a change in practice, but an improvement in quality of services offered to STEMI patients on the non-interventional site. Much depends on the style of leadership within the department and whether cross-departmental involvement is acceptable to key stakeholders. A transformational style of leadership fosters a collaborative environment where all stakeholders feel that views can be expressed democratically and without fear of criticism and can be integrated with the views of others.

\section{Conclusion}

Factors influencing delay to treatment for time-critical conditions need to be carefully monitored. Each stage of the patient journey requires scrutiny to assess whether acceptable times are achieved or whether changes need to be made to effect improvement. One department carrying out this kind of audit can inform other services also looking at different time-critical conditions. The data collected from the STEMI patients transferring for PPCI has informed the Stroke thrombolysis service and enabled decisions to be made regarding the best way of delivering that service. It is useful to have an individual with a particular interest and input into monitoring and auditing targets to process map the patient journey and flag up and address any areas of concern. This person may be able to engage others, especially in the first phase of system change.

It is impossible to calculate the financial savings of this simple intervention. Some patients who have prolonged FMC-B times will have left ventricular dysfunction caused by loss of viable myocardium. The intervention is thought to be effective in ensuring rapid FMC-B times and therefore reducing the incidence of morbidity and mortality from heart failure.

\section{References}

1. Erhardt L, Herlitz J, Bossaert L et al ESC Guidelines for the management of acute coronary syndromes in patients presenting without persistent ST-segment elevation eurheartj. 2011, 32: $2999-3054$

2. Treatment of Heart Attack National Guidance Final Report of the National Infarct Angioplasty Project (NIAP) 2008 www.bcis.org.uk/resources/documents/NIAP\%20Final\%20R eport.pdf accessed 12/1/2009

3. Emergency triage Manchester Triage Group. MackwayJones K, ed. 1997 London: BMJ Publishing Group

4. Cooke M, Jinks S. Does the Manchester triage system detect the critically ill? J Accid Emerg Med 1999;16:179-181

5. van der Wulp I, van Baar ME, Schrijvers AJ. Reliability and validity of the Manchester Triage System in a general emergency department patient population in the Netherlands: results of a simulation study. Emerg Med J. 2008 25;431-4

6. College of Emergency Medicine Clinical Standards for Emergency Departments 2011 www.collemergencymed.ac.uk/ShopFloor/Clinical\%20Standards accessed 12/11/11

\section{Declaration of interests}

There is no conflict of interest to declare

\section{Acknowledgements}

John Gray, Paul Curran, Bernie Hillen, Linsey Sheerin, nursing and medical records staff of the ED. 